\title{
Basic Relevant Theories for Combinators at Levels I and II
}

\author{
Koushik PaL \\ Department of Mathematics, \\ Indian Institute of Technology Kanpur \\ kapal@iitk.ac.in \\ Robert K. Meyer \\ Automated Reasoning Group, RSISE, \\ Australian National University \\ Bob.Meyer@anu.edu.au \\ Received by Martin Bunder \\ Published July 8, 2005 \\ http://www.philosophy.unimelb.edu.au/ajl/2005
}

(c) 2005 Koushik Pal and Robert K. Meyer

\begin{abstract}
The system $\mathrm{B}+$ is the minimal positive relevant logic. $\mathrm{B}+$ is trivially extended to $B+T$ on adding a greatest truth (Church constant) $T$. If we leave $\vee$ out of the formation apparatus, we get the fragment $B \wedge T$. It is known that the set of $A L L B \wedge T$ theories provides a good model for the combinators $C L$ at Level-I, which is the theory level. Restoring $\vee$ to get back B+T was not previously fruitful at Level-I, because the set of all $\mathrm{B}+\mathrm{T}$ theories is NOT a model of CL. It was to be expected from semantic completeness arguments for relevant logics that basic combinator laws would hold when restricted to PRIME B+T theories. Overcoming some previous difficulties, we show that this is the case, at Level I. But this does not form a model for $\mathrm{CL}$. This paper also looks for corresponding results at Level-II, where we deal with sets of theories that we call propositions. We adapt work by Ghilezan to note that at Level-II also there is a model of $C L$ in $B \wedge T$ propositions. However, the corresponding result for $B+T$ propositions extends smoothly to Level-II only in part. Specifically, only some of the basic combinator laws are proved here. We accordingly leave some work for the reader.
\end{abstract}

\section{INTRODUCTION}

This paper is an essay at an intersection of Philosophy, Computer Science and Mathematics. That intersection, for present purposes, we take to be Logicand in particular relevant and other substructural logics. It is also an essay in 
Levels-in what happens when we climb a set-theoretic level or two in order to enrich a supply of formal objects with extra ones. It is thus, for example, that the collection of real numbers was placed on a "sound" basis by igth and early 20 th century researchers.

EXAMPLE I. $\mathfrak{R}$ AT LEVEL-I Consider the problem of locating $\pi$ among the set $\mathfrak{Q}$ of rational numbers. In $\mathfrak{Q}$, we find 3, 3.1, 3.14 and so forth, but none of these successive "finite approximations" is equal to the infinitely long "decimal expansion of $\pi$ ". Idea: Let's extend our usual arithmetic on $\mathfrak{Q}$ to define also arithmetic operations on (properly chosen) subsets of $\mathfrak{Q}$. Such a subset of $\mathfrak{Q}$ will be an ideal (number). And among those ideals will be $\pi$, with its usual arithmetical properties.

Here's the basic idea: We enrich a Level-o collection $\mathfrak{C}$ of formal objectsin this case $\mathfrak{Q}$-by passing to a Level-I collection of (appropriately chosen) subsets of $\mathfrak{C}$. Some of these subsets (the principal ideals, in the $\mathfrak{Q}$ case) may be taken as the representatives at Level-I of the original Level-o entities. As for the new entities arising at Level-I (in the sample case, the non-principal ideals corresponding to Dedekind cuts, which are the other real numbers), the magic of set-theoretic ascent gives them the right properties to be the rest of $\Re$.

EXAMPLE 2. BOOle AT LEVEL-I I There is no good reason to stop at Level-I, either. Consider now the case of Boolean algebras, under Stone representation. Everybody knows that Boolean algebra is in some appropriate sense the algebra of sets. But what that sense is became crystal clear only with the work of Stone [II]. We take (with some redundancy) a Boolean algebra to be a structure $\mathcal{B}=\langle\mathrm{B}, \neg, \wedge, \vee, \top, \perp\rangle$, where $\mathrm{B}$ is a set closed under complement $(\neg)$, meet $(\wedge)$ and join $(\vee)$, and $T$ and $\perp$ are respectively the top and the bottom of the algebra under the induced ordering. An ultrafilter $U$ in $\mathcal{B}$ will be any subset of $B$ satisfying, for all $a, b$ in $B$,

[T^] $a \wedge b \in U$ iff $a \in U$ and $b \in U$

$[\mathrm{T} \neg] \neg \mathrm{a} \in \mathrm{U}$ iff $\mathrm{a} \notin \mathrm{U}$.

Note that, from the (Level-o) viewpoint of an element $b$ of $B$, an ultrafilter is at Level-I. But Stone's Representation Theorem, in representing B, takes us already to Level-II, on the following recipe:

- $\mathrm{UB}={ }_{\mathrm{df}}\{\mathrm{U}: \mathrm{U}$ is an ultrafilter in $\mathrm{B}\}$

- $P U B={ }_{d f}\{W: W \subseteq U B\}$, the power set of $U B$

- $\mathcal{P U B}=\langle\mathrm{PUB}, \neg, \cap, \cup, \emptyset, \mathrm{UB}\rangle$ is a Boolean set algebra

where, for any sets $\alpha, \beta$ of ultrafilters in $B, \alpha \wedge \beta$ and $\alpha \vee \beta$ are respectively the intersection and union, and $\neg \alpha$ the complement relative to UB, of the sets. As for $\perp$ and $T$, they are respectively the null set and the universal set of 
ultrafilters in B. The utility of the ascent to PUB is that there is an isomorphic copy of $B$ itself two levels up. For let an embedding $h: \mathcal{B} \rightarrow \mathcal{P U B}$ be defined, by assigning to every element $b$ the set of ultrafilters in $\mathcal{B}$ to which it belongs. i.e., for $b \in B$, fix

$[\mathrm{Dh}] \mathrm{h}(\mathrm{b})=\{\mathrm{U}: \mathrm{b} \in \mathrm{U}$ and $\mathrm{U} \in \mathrm{UB}\}$

Thus every Boolean algebra $\mathcal{B}$ finds a home away from home in a $\mathcal{P U} \mathcal{B}$.

EXAMPLE 3. RELEVANT LOGiCS Structures may be enriched and completed, as we have just recalled, by raising Levels. We now turn to relevant logics. They have come, semantically, a long way. Beginning in [Io] from the Orlov-MohChurch-Anderson-Belnap system R of relevant implication, Routley, Meyer and others produced a series of articles on the Semantics of Entailment, based on Kripke-style ternary relational postulates. In [9] such postulates were found, in the $\langle\rightarrow, \wedge, \vee\rangle$ vocabulary, for the Anderson-Belnap systems $R+$ of positive relevant implication, $\mathrm{E}+$ of positive entailment, and $\mathrm{T}+$ of positive ticket entailment. Among these positive relevant logics there was a new and natural minimal one, which we called $\mathrm{B}+$.

We concentrate, in this paper, on $B+$, and on the fragments, alternative formulations and conservative extensions that make up what we may call the $B+$ family of minimal logics. We will be concerned, in our work with the $B+$ family, with collections of formal objects at Levels I and II. At Level-I our focus is on theories-intuitively, sets of sentences that are logically closed. We concentrate at Level-II on what we call proposition:-special collections of theories.

EXAMPle 4. COMBinatory LOGiC AND $\lambda$-Calculi Our next topic will be the Combinatory Logic CL of Curry and Feys $[3]$. There is a delightful coincidence between axiom candidates for various relevant logics and the combinators of which, on the analysis of Curry, these candidate axioms were (in Curryspeak) the "functional characters" (nowadays "types"). In fact the coincidence not only reflected Curry but improved him; some combinators untypeable by Curry correspond anyway to famous theorems of logic, which have been embraced (and repudiated) with vigor.

Twenty years ago, this coincidence was independently rediscovered and deepened by researchers in $\lambda$-calculus; specifically, by Dezani and her colleagues in [I] and elsewhere. They added intersection types to Curry's arrow types. (Add with [5] type intersection for logical $\wedge$ to Curry's original function type for logical $\rightarrow$, and behold the improvement.) As relevant semantical analysis predicted, further combinators (sample: $W^{*}$, equivalently WI, SII, or $\lambda x . x x$ ) have

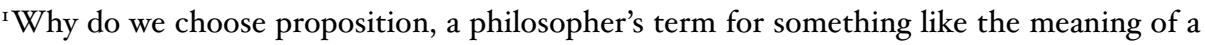
sentence? We have in mind the so-called UCLA view, on which a proposition is explicated as the set of possible worlds in which a sentence is true. Reduced to its (Level-II) syntactical residue, this is the set of special theories-namely, the classically consistent and complete ones-to which the sentence belongs. We generalize!
} 
non-trivial types in the enriched setup. And the structure of these types is that conferred by the analysis of conjunction $\wedge$ and implication $\rightarrow$ in the minimal relevant logic $\mathrm{B}+$ of [9].

So exciting are the connections between $C L$ and relevant logics that we have dubbed them The Key to the Universe. There is already good evidence that the key fits because there are the semantical completeness proofs themselves. Here, we shall insert that key a little further into its lock. We look again at how to account for disjunction $\vee$ in modeling CL. For $\vee$ (with its usual truthfunctional semantics) has been an ingredient in $\mathrm{B}+$ and other relevant logics from the beginning. Dezani, Meyer and Motohama did offer a $B+T$ model of $\lambda$ and $\mathrm{CL}$ in [5], appealing to (so-called) Harrop theories. A principal result here is a better fit with the semantical and logical intuitions of [9]. We look to prime $\mathrm{B}+\mathrm{T}$-theories in general, and not just the Harrop subclass thereof, as the appropriate vehicle with which to make the laws governing primitive $\mathrm{CL}$ combinators true. But Dezani and her colleagues, for their part, are not to be denied. For it was (what we call) the Better Bubbling Lemma (henceforth, ввL) of Dezani et al. in [6] that led to our new verification of the primitive combinatory equations in prime $\mathrm{B}+\mathrm{T}$ theories.

In this paper, we follow $[\overline{8}$ to move from Level-I to Level-II to find systems which model $\lambda$ and hence CL. Levels arise out of the way one looks at systems. We discuss, as anticipated above, three levels. (You can have more, if you like.) Level-o is that of the elements of a given logical algebra-typically, an Algebra of Formulas, where each element is a Well-Formed-Formula (w FF) 2 For present purposes, at Level-I each element is a theory, which is a collection of WFFs with some nice properties like closure under conjunction and entailment. (For technical reasons involving the relevantly irrelevant combinator $\mathrm{K}$ and other cancellators, we follow [5] by requiring the theories of this paper to be non-empty.) At Level-II we have propositions, which are collections of theories with further nice properties. (We trust that theories and propositions will become more clear as we go along.)

We recall the definition of $B \wedge T$ as a relational system from [5]. $B \wedge T$ is little more than a fragment of $B+$. In presenting $B \wedge T$, we had a choice between an assertional and a relational formulation 3 This is a distinction without much of a difference, since to assert $A \rightarrow B$ as a theorem of logic comes sensibly to the same thing (when all the "i"s are dotted and "t"s are crossed) as to claim that $A$ logically entails B.4

\footnotetext{
${ }^{2}$ We allow some ambiguity in specifying Level-o objects. This leaves open the possibility, as in the Boolean algebra example above, that distinct w FFs have been identified via an appropriate quotient construction. Think in this case of Level-o objects as congruence classes of wFF.

${ }^{3}$ We are even-handed here, formulating the richer $\mathrm{B}+\mathrm{T}$ below as an assertional system.

${ }^{4}$ More specifically, we may borrow from Curry $[4]$. The w FFs (Curry's oBs) of a propositional logic are the Level-o objects built up from atoms (propositional variables and constants) via the primitive operations ( $\rightarrow$ and the like). The elementary statements are then formed by attaching predicators to wFFs. To formulate a system assertionally is to choose a I-place predicator (say $\vdash$ ) and to insist that the elementary statements (and hence the theorems) are things of the form
} 
$\mathrm{B} \wedge \mathrm{T}$ is the $\langle\rightarrow, \wedge\rangle$ fragment of $\mathrm{B}+$, enriched with a greatest truth, given by Church constant T. Upper-case ' $A$ ', 'B', etc. are used to denote syntactical variables. $\leqslant$ is a binary predicate in the language, which is read as 'entails'. With these we state the formal definition of the $B \wedge T$ system. The axioms for the $\mathrm{B} \wedge \mathrm{T}$ system are as follows:

$$
\begin{array}{ll}
\text { Reflex. } & A \leqslant A \\
\text { Top. } & A \leqslant T \\
\text { Top } \rightarrow . & T \leqslant(T \rightarrow T) \\
\text { Idem } \wedge . & A \leqslant(A \wedge A) \\
\wedge \mathrm{E} . & (A \wedge B) \leqslant A,(A \wedge B) \leqslant B \\
\rightarrow \wedge \text { I. } & (A \rightarrow B) \wedge(A \rightarrow C) \leqslant(A \rightarrow(B \wedge C))
\end{array}
$$

The rules of the system are as follows. (Note that $\Rightarrow$ has been used as a metalogical connective in framing rules.)

$$
\begin{array}{ll}
\text { Trans } \wedge . & A \leqslant B \leqslant C \Rightarrow A \leqslant C \\
\text { Mon } \wedge . & A \leqslant A^{\prime}, B \leqslant B^{\prime} \Rightarrow A \wedge B \leqslant A^{\prime} \wedge B^{\prime} \\
\text { Mon } \rightarrow . & A^{\prime} \leqslant A, B \leqslant B^{\prime} \Rightarrow A \rightarrow B \leqslant A^{\prime} \rightarrow B^{\prime}
\end{array}
$$

Our first task now is to prove that the results at Level-I extend smoothly to Level-II for $\mathrm{B} \wedge \mathrm{T}$ theories. The idea of moving to Level-II is for some obvious reasons which will be made clear soon. But first we start with some definitions and theorems and prove that the results for $\mathrm{B} \wedge \mathrm{T}$ theories hold at Level-II also.

Definition i (THEORY) A THEORY or B^T-THEORY is a non-empty set of formulas closed under conjunction and $\mathrm{B} \wedge \mathrm{T}$-entailment.

Definition 2 (A-Theory) An A-theory (denoted “ $A \uparrow$ ”) is a theory containing the formula $A$ and all the formulas $C$ that are $B \wedge T$-entailed by $A$, i.e., $A \uparrow=\{C: A \leqslant C\}$.

\section{THEOREM I The intersection of two theories is a theory.}

Proof Let $T_{1}$ and $T_{2}$ be two theories. So, $T_{1} \neq \emptyset \neq T_{2}$. (i) Suppose $A \in T_{1} \cap T_{2}$ and $A \leqslant B$. It follows that $A \in T_{1}$ and $A \leqslant B$, so $B \in T_{1}$ (since $T_{1}$ is a theory). Similarly, $B \in T_{2}$. Hence, $B \in T_{1} \cap T_{2}$. (ii) Suppose $A, B \in T_{1} \cap T_{2}$. So, $A, B \in T_{1}$ and thus $A \wedge B \in T_{1}$ (since $T_{1}$ is a theory), and similarly, $A \wedge B \in T_{2}$. Hence, $A \wedge B \in T_{1} \cap T_{2}$. (iii) $T \in T_{1}$ and $T \in T_{2}$, so, $T \in T_{1} \cap T_{2}$. So, $T_{1} \cap T_{2} \neq \emptyset$. Hence, $T_{1} \cap T_{2}$ is a theory.

$\vdash$ A. To formulate a system relationally is to choose a 2-place predicator (say $\leqslant$ ) and to insist that the elementary statements (and hence the theorems) are things of the form $A \leqslant B$. 
Definition 3 (proposition) A B $\wedge$ T-proposition P is a non-empty set of theories closed under sub-theory relation and intersection. That is (i) if $T_{1}$ and $T_{2}$ are two theories, $T_{1} \subseteq T_{2}$ and $T_{1} \in P$, then $T_{2} \in P$, and (ii) if $T_{1} \in P$ and $\mathrm{T}_{2} \in \mathrm{P}$, then $\mathrm{T}_{1} \cap \mathrm{T}_{2} \in \mathrm{P}$.

Definition 4 (A-proposition) An A-proposition (written "A”, and also called the principal proposition for the formula $A$ ) is the proposition consisting of $A \uparrow$ and all of its its super-theories (it is of course closed under intersection).

In what immediately follows, we will call a $B \wedge T$ theory simply a theory; $B \wedge T$ proposition, a proposition; and $\mathrm{B} \wedge \mathrm{T}$ entailment, entailment. We will also use $T_{1}, T_{2}$, etc. to denote theories, $P, Q$, etc. to denote propositions, $\underline{A}, \underline{B}$, etc. to denote principal propositions for formulas $A, B$, etc., and $A, B$, etc. to denote well formed formulas. Notice that by the axioms of $B \wedge T, T$ and $T \rightarrow T$ belong to every theory of $\mathrm{B} \wedge \mathrm{T}$. The propositions, on the other hand, are by definition non-empty. We take our next definition from [8].

Definition 5 (open sets) A set $\Theta$ is said to be open if (i) if $\alpha \in \Theta$ and $\alpha \subseteq \beta$ then $\beta \in \Theta$, and (ii) If $\bigcup_{i \in I} \alpha_{i} \in \Theta$ then $\exists i_{0} \in$ I such that $\alpha_{i_{0}} \in \Theta$.

Theorem 2 An A-Theory is the minimum theory containing A.

Proof Suppose $T_{1}$ is a theory containing $A$. Since $T_{1}$ is a theory, it is closed under conjunction and entailment relation. Therefore, for all formulas $B$ where $\mathrm{A} \leqslant \mathrm{B}, \mathrm{B} \in \mathrm{T}_{1}$. Hence, $\mathrm{A} \uparrow \subseteq \mathrm{T}_{1}$.

Theorem 3 An A-proposition is the minimum proposition containing $A \uparrow$.

Proof Suppose $P$ is a proposition containing $A \uparrow$. Since $P$ is a proposition, it is closed under sub-theory relation and intersection. Therefore, all theories of which $A \uparrow$ is a sub-theory, i.e., all super-theories of $A \uparrow$ belong to $P$ and hence also their intersections. Hence, $\underline{A} \subseteq \mathrm{P}$.

Theorem 4 Any principal proposition is open.

Proof (i) Let $T_{1} \in \underline{A}$ and $T_{1} \subseteq T_{2}$. So $T_{2} \in \underline{A}$ (since $\underline{A}$ is closed under subtheory relation). (ii) Suppose that $\bigcup_{i \in I} \alpha_{i} \in \underline{A}$ (each $\alpha_{i}$ is a theory). It follows that $A \uparrow \subseteq \bigcup_{i \in I} \alpha_{i}$, and hence $A \in \bigcup_{i \in I} \alpha_{i}$, and it follows that there is some $i_{0} \in$ I where $A \in \alpha_{i_{0}}$. So, $\exists i_{0} \in$ I such that $A \uparrow \subseteq \alpha_{i_{0}}$ (since $\alpha_{i_{0}}$ is a theory), and it follows that $\exists i_{0} \in I$ such that $\alpha_{i_{0}} \in \underline{A}$. Hence, any principal proposition is open.

Theorem 5 Any theory $\mathrm{T}_{1}=\bigcup\left\{\mathrm{B} \uparrow: \mathrm{B} \in \mathrm{T}_{1}\right\}$.

Proof Let $B \in T_{1}$. We have $B \in B \uparrow$, so $B \in \bigcup\left\{B \uparrow: B \in T_{1}\right\}$. Conversely, suppose $C \in \bigcup\left\{B \uparrow: B \in T_{1}\right\}$. So, there is some $B \in T_{1}$ such that $C \in B \uparrow$. Since $T_{1}$ is a theory and $B \in T_{1}$, it follows that $B \uparrow \subseteq T_{1}$ (by Theorem 2). So, $C \in T_{1}$, and hence, $T_{1}=\bigcup\left\{B \uparrow: B \in T_{1}\right\}$. 
Theorem 6 For principal proposition $\underline{\mathrm{A}}, \underline{\mathrm{A}}=\bigcup\{\underline{\mathrm{B}}: \mathrm{B} \uparrow \in \underline{\mathrm{A}}\}$.

Proof Consider $\underline{B}$ such that $B \uparrow \in \underline{A}$. Since $\underline{B}$ is the smallest proposition containing $B \uparrow, \underline{B}$ and so $\bigcup\{\underline{B}: B \uparrow \in \underline{A}\}$ is a subset of $\underline{A}$. Conversely suppose $T_{1} \in$ A. Clearly, $\mathrm{T}_{1} \neq \emptyset$. Now $\mathrm{T}_{1}=\bigcup\left\{\mathrm{C} \uparrow: \mathrm{C} \in \mathrm{T}_{1}\right\}$ and so $\bigcup\left\{\mathrm{C} \uparrow: \mathrm{C} \in \mathrm{T}_{1}\right\} \in \underline{\mathrm{A}}$. But $\underline{\mathrm{A}}$ is open and $C \uparrow$ is a theory for each $C$. Hence there is a $C \in T_{1}$ such that $C \uparrow \in \underline{A}$. Also, $\mathrm{C} \uparrow \subseteq \mathrm{T}_{1}$ (by Theorem 2). Therefore $\mathrm{T}_{1} \in \underline{\mathrm{C}}$, and so, $\mathrm{T}_{1} \in \bigcup\{\underline{C}: \mathrm{C} \uparrow \in \underline{\mathrm{A}}\}$. Therefore, $\underline{A} \subseteq \bigcup\{\underline{B}: \mathrm{B} \uparrow \in \underline{A}\}$, and hence, $\underline{A}=\bigcup\{\underline{B}: \mathrm{B} \uparrow \in \underline{A}\}$.

THEOREM 7 The intersection of two propositions is always non-empty.

Proof Suppose $\mathrm{P}$ and $\mathrm{Q}$ are two propositions. By definition, $\mathrm{P} \neq \emptyset \neq \mathrm{Q}$. So, there are $T_{1}, T_{2}$ such that $T_{1} \in P$ and $T_{2} \in Q$. Now, $T_{1}=\bigcup\left\{C \uparrow: C \in T_{1}\right\}$, and $T_{2}=\bigcup\left\{D \uparrow: D \in T_{2}\right\}$. Define $T_{3}=\left\{E: \exists C \in T_{1}\right.$ and $\exists D \in T_{2}$ where $\left.C \wedge D \leqslant E\right\}$. Clearly, $T_{1} \subseteq T_{3}$ and $T_{2} \subseteq T_{3}$. Therefore, $T_{3} \in P$ and $T_{3} \in$ Q, i.e., $T_{3} \in P \cap Q$. We have shown that $\mathrm{P} \cap \mathrm{Q}$ is non-empty.

THEOREM 8 The intersection of two propositions is always a proposition.

Proof Let $\mathrm{P}$ and $\mathrm{Q}$ be two propositions. By Theorem 6, $\mathrm{P} \cap \mathrm{Q} \neq \emptyset$. (i) Suppose $T_{1} \in P \cap Q$ and $T_{1} \subseteq T_{2}$. Then $T_{1} \in P$ and $T_{1} \subseteq T_{2}$ and $P$ is a proposition. Therefore, $T_{2} \in P$. Similarly $T_{2} \in Q$. And hence $T_{2} \in P \cap Q$. (ii) Suppose that $T_{1}$ and $T_{2} \in P \cap Q$. Then $T_{1}$ and $T_{2} \in P$ and so $T_{1} \cap T_{2} \in P$. Similarly, $\mathrm{T}_{1} \cap \mathrm{T}_{2} \in \mathrm{Q}$. And hence $\mathrm{T}_{1} \cap \mathrm{T}_{2} \in \mathrm{P} \cap \mathrm{Q}$. Therefore, $\mathrm{P} \cap \mathrm{Q}$ is closed under the sub-theory relation and under intersection, and is non-empty. It follows that it is a propositon.

THEOREM 9 The intersection of two principal propositions is a principal proposition.

Proof Suppose that $\underline{A}$ and $\underline{B}$ are two principal propositions. Now, $\underline{A}=\left\{T_{1}\right.$ : $\left.A \uparrow \subseteq T_{1}\right\}$ and $\underline{B}=\left\{T_{2}: B \uparrow \subseteq T_{2}\right\}$. We have already proved that $\underline{A} \cap \underline{B}$ is a proposition. So now we need to show that it is a principal proposition. Claim: $\underline{A} \cap \underline{B}=\underline{A} \wedge \mathrm{B}$. Let $T_{1} \in \underline{A} \cap \underline{B}$ This holds if and only if $A \uparrow \subseteq T_{1}$ and $B \uparrow \subseteq T_{1}$ which, in turn, holds if and only if $A \in T_{1}$ and $B \in T_{1}$, if and only if $A \wedge B \in T_{1}$ (since, $T_{1}$ is a theory). This is equivalent to $(A \wedge B) \uparrow \subseteq T_{1}$ (by Theorem 5), and hence $T_{1} \in(A \wedge B)$. The intersection of two principal propositions is a principal proposition.

\section{B $A T$ IS A MODEL FOR COMBINATORS AT LEVEL-I}

The idea of a model for combinators at a Level is that the elements on which the combinators operate are elements of that particular Level. The defining equation for any combinator also depends on the Level at which we work. For example, at Level-I, we assign a theory to each combinator or other combinatorial term. But at Level-II, we assign propositions to combinators. And then we show that, where $t=u$ is a provable equation of $C L$, both $t$ and $u$ must be assigned the same object. The larger part of this task is to show that the 
defining equations for particular combinators turn out true.5 But it is meet also to show that we have so fashioned our semantic apparatus that the rules of inference ${ }^{6}$ of $C L$ are truth-preserving.

We will look at defining equations for three combinators in particular here, namely, I, K and $\mathrm{W}^{*}$. For these combinators, we will prove that the defining equations turn out true in $\mathrm{B} \wedge \mathrm{T}$-theories. Proofs for the other combinators are similar and hence are omitted here. Let's start with the definition of the fusion operator at different Levels and a basic lemma.

Definition 6 (FUSION OPERATOR AT LEVEL-O) We can introduce the fusion operator ' $\circ$ ' at Level-o by just including it in our vocabulary as one more sentential connective, together with a "residuation" rule. While [9] shows that this produces conservative extensions of standard relevant logics (including those of this paper), we resist that course here.

Definition 7 (Fusion at Level-i $\left(\circ_{1}\right)$ ) Let $\mathrm{T}_{1}$ and $\mathrm{T}_{2}$ be two theories. Then $\mathrm{T}_{1} \circ_{1} \mathrm{~T}_{2}$ is defined as

$$
\mathrm{T}_{1} \circ_{1} \mathrm{~T}_{2}=\left\{\mathrm{B}: \exists \mathrm{A} \text { where } \mathrm{A} \rightarrow \mathrm{B} \in \mathrm{T}_{1} \text { and } \mathrm{A} \in \mathrm{T}_{2}\right\}
$$

Definition 8 (Fusion at Level-i $\left(\mathrm{o}_{2}\right)$ ) Let $\mathrm{P}$ and $\mathrm{Q}$ be two propositions. Then $\mathrm{P} \mathrm{O}_{2} \mathrm{Q}$ is defined as

$$
\mathrm{P} \circ_{2} \mathrm{Q}=\left\{\mathrm{T}_{1}: \exists \mathrm{T}_{2} \in \mathrm{P} \text { and } \exists \mathrm{T}_{3} \in \mathrm{Q} \text { where } \mathrm{T}_{2} \circ_{1} \mathrm{~T}_{3} \subseteq \mathrm{T}_{1}\right\}
$$

Lemma io The fusion of two theories is also a theory.

Proof Let $T_{1}$ and $T_{2}$ be two theories. (i) $T \rightarrow T \in T_{1}$ and $T \in T_{2}$. Therefore, $T \in T_{1} \circ_{1} T_{2}$ and hence, $T_{1} \circ_{1} T_{2}$ is non-empty. (ii) Suppose $A \in T_{1} \circ_{1} T_{2}$ and $A \leqslant B$. So $\exists C$ such that $C \rightarrow A \in T_{1}$ and $C \in T_{2}$. But $C \leqslant C$ and $A \leqslant B \Rightarrow C \rightarrow A \leqslant C \rightarrow B$. Hence, $C \rightarrow B \in T_{1}$ (as $T_{1}$ is a theory). Therefore, $B \in T_{1} \circ_{1} T_{2}$. (iii) Suppose $A, B \in T_{1} \circ_{1} T_{2}$. Therefore $(\exists C$ where $C \rightarrow A \in$ $\mathrm{T}_{1}$ and $\left.\mathrm{C} \in \mathrm{T}_{2}\right)$ and $\left(\exists \mathrm{D}\right.$ where $\left.\mathrm{D} \rightarrow \mathrm{B} \in \mathrm{T}_{1} \& \mathrm{D} \in \mathrm{T}_{2}\right)$. Now, $\mathrm{C} \wedge \mathrm{D} \leqslant \mathrm{C}$ and $A \leqslant A \Rightarrow C \rightarrow A \leqslant C \wedge D \rightarrow A$. Therefore, $C \wedge D \rightarrow A \in T_{1}$. (since $T_{1}$ is a theory) Similarly, $C \wedge D \rightarrow B \in T_{1}$. Therefore, $C \wedge D \rightarrow A \wedge B \in T_{1}$. But, $C \wedge D \in T_{2}$ (since $T_{2}$ is closed under conjunction). Hence, $A \wedge B \in T_{1} \circ_{1} T_{2}$. Thus, $T_{1} \circ T_{2}$ is non-empty and also closed under conjunction and entailment. Hence it is a theory.

COROLLARY II The fusion of a finite number of theories is also a theory.

Proof Use induction and Lemma io.

5These are axioms like $I x=x, W x y=x y y$, etc.

${ }^{6}$ For example, as found in [3]. 
Now we will prove that $\mathrm{B} \wedge \mathrm{T}$-theories model the three combinators mentioned above. Here are defining equations for some combinators.

$$
\begin{aligned}
\mathrm{Ix} & =x \\
\mathrm{~K} x y & =x \\
\mathrm{C} x y z & =x z y \\
\mathrm{C}^{*} x y & =y x \\
\mathrm{~B} x y z & =x(y z) \\
\mathrm{B}^{\prime} x y z & =y(x z) \\
\mathrm{S} x y z & =x z(y z) \\
\mathrm{W}^{\prime} y & =x y y \\
\mathrm{~W}^{*} x & =x x
\end{aligned}
$$

We assign a particular theory to each combinator. To indicate the assignment succinctly, we enclose a formula scheme in brackets. The associated theory $\mathrm{T}$ is then the smallest set of formulas that contains all conjunctions of one or more instances of the scheme and which is closed under the entailment relation $\leqslant$. Here are the definitions.

$$
\begin{aligned}
& \mathrm{I}=[\mathrm{A} \rightarrow \mathrm{A}] \\
& =\left\{C \text { : for some finite set } M \text { and formulas } A_{m}\right. \text {, } \\
& \mathrm{K}=[\mathrm{A} \rightarrow(\mathrm{B} \rightarrow \mathrm{A})] \\
& \left.\bigwedge_{m \in M}\left(A_{m} \rightarrow A_{m}\right) \leqslant C\right\} \\
& C=[(A \rightarrow(B \rightarrow C)) \rightarrow(B \rightarrow(A \rightarrow C))] \\
& C^{*}=[A \rightarrow((A \rightarrow B) \rightarrow B)] \\
& B=[(A \rightarrow B) \rightarrow((C \rightarrow A) \rightarrow(C \rightarrow B))] \\
& \mathrm{B}^{\prime}=[(\mathrm{A} \rightarrow \mathrm{B}) \rightarrow((\mathrm{B} \rightarrow \mathrm{C}) \rightarrow(\mathrm{A} \rightarrow \mathrm{C}))] \\
& S=[(A \rightarrow(B \rightarrow C)) \rightarrow((A \rightarrow B) \rightarrow(A \rightarrow C))] \\
& W=[(A \rightarrow(A \rightarrow B)) \rightarrow(A \rightarrow B)] \\
& W^{*}=[((A \rightarrow B) \wedge A) \rightarrow B]
\end{aligned}
$$

The definitions for the theories assigned to each of the combinators are similar to that for I. For example, a formula $\mathrm{D}$ belongs to the set $\mathrm{K}$ iff there is some conjunction $C$ of formulas of the form $A \rightarrow(B \rightarrow A)$ such that $C \leqslant D$. Here we show that the set $\mathrm{I}$ is indeed a theory. Proofs for other combinators are similar.

Claim I2 $[A \rightarrow A]$ is a theory.

Proof (i) $[A \rightarrow A]$ contains all instances of the formulas of the kind $A \rightarrow$ A. So it is surely non-empty. (ii) Suppose $C, D \in[A \rightarrow A]$. Then $\exists M, N$ where $\bigwedge_{m \in M}\left(A_{m} \rightarrow A_{m}\right) \leqslant C$ and $\bigwedge_{n \in N}\left(A_{n} \rightarrow A_{n}\right) \leqslant D$. Thus we have $\bigwedge_{i \in M \cup N}\left(A_{i} \rightarrow A_{i}\right) \leqslant C \wedge D$ and hence $C \wedge D \in[A \rightarrow A]$. (iii) Suppose $C \in[A \rightarrow A]$ and $C \leqslant D$. Then $\exists M$ where $\bigwedge_{m \in M}\left(A_{m} \rightarrow A_{m}\right) \leqslant C \leqslant D$. Thus $D \in[A \rightarrow A]$. Thus $[A \rightarrow A]$ is non-empty and closed under conjunction and entailment. Hence, it is a theory. 
We come now to the famous Bubbling Lemma ( $\mathrm{BL}$ ), which was a principal weapon invoked in [I] to show that filters on intersection types provide a model of $\lambda$. It will play the same role here for us, changing only the vocabulary to say that $\mathrm{B} \wedge \mathrm{T}$-theories provide a model for combinators.

Lemma I3 (bubbling lemma) Assume it is not the case that $\mathrm{D} \equiv \mathrm{T}$. Assume moreover that $\bigwedge_{i \in \mathrm{I}}\left(A_{i} \rightarrow B_{i}\right) \leqslant C \rightarrow D$ for some finite non-empty index set $\mathrm{I}$. Then there is a finite non-empty subset $\mathrm{J}$ of I such that

$$
C \leqslant \bigwedge_{j \in J} A_{j} \text { and } \bigwedge_{j \in J} B_{j} \leqslant D .
$$

Proof Proof given in [I]. (Note that $\equiv$ is defined by setting $A \equiv B={ }_{d f} A \leqslant$ $B$ and $B \leqslant A$.)

Claim I4 $\mathrm{I}_{1} \mathrm{~T}_{1}=\mathrm{T}_{1}$

Proof Let $A \in \mathrm{I}_{1} \mathrm{~T}_{1}$. Then $\exists \mathrm{B}$ where $\mathrm{B} \rightarrow \mathrm{A} \in \mathrm{I}$ and $\mathrm{B} \in \mathrm{T}_{1}$. But $\mathrm{B} \rightarrow A \in \mathrm{I}$ means that there is a finite $M$ where $\bigwedge_{k \in M}\left(A_{k} \rightarrow A_{k}\right) \leqslant B \rightarrow A$. Therefore, by the Bubbling Lemma, $B \leqslant \bigwedge_{j \in J} A_{j}$ and $\bigwedge_{j \in J} A_{j} \leqslant A$ for some $J \subseteq M$. Since $B \in T_{1}$ and $T_{1}$ is a theory, it follows that $\bigwedge_{j \in J} A_{j} \in T_{1}$. And hence, $A \in T_{1}$ (for similar reasons.). So, $\mathrm{I}_{1} \mathrm{~T}_{1} \subseteq \mathrm{T}_{1}$.

Conversely, suppose $A \in T_{1}$. Since $A \rightarrow A \in I$ (by definition), it follows that $A \in \mathrm{I} \circ_{1} \mathrm{~T}_{1}$. So, $\mathrm{T}_{1} \subseteq \mathrm{I} \circ_{1} \mathrm{~T}_{1}$. Hence, $\mathrm{I} \circ_{1} \mathrm{~T}_{1}=\mathrm{T}_{1}$.

Claim $15 \quad \mathrm{~K}_{1} \mathrm{~T}_{1} \circ_{1} \mathrm{~T}_{2}=\mathrm{T}_{1}$

Proof Suppose $A \in K \circ_{1} T_{1} \circ_{1} T_{2}$. Then $\exists B, C$ such that $B \rightarrow(C \rightarrow A) \in$ $K, B \in T_{1}$ and $C \in T_{2}$. But $B \rightarrow(C \rightarrow A) \in K$, so $\exists$ finite I such that $\bigwedge_{i \in I}\left(D_{i} \rightarrow\right.$ $\left.\left(E_{i} \rightarrow D_{i}\right)\right) \leqslant(B \rightarrow(C \rightarrow A))$. By the Bubbling Lemma, $\exists J \subseteq$ I such that $B \leqslant$ $\bigwedge_{j \in J} D_{j}$ and $\bigwedge_{j \in J}\left(E_{j} \rightarrow D_{j}\right) \leqslant C \rightarrow A$ Again by Bubbling Lemma, $\exists M \subseteq$ J such that $C \leqslant \bigwedge_{k \in M} E_{k}$ and $\bigwedge_{k \in M} D_{k} \leqslant A$. Now, $B \in T_{1}$ and $T_{1}$ is a theory $\Rightarrow \bigwedge_{j \in J} D_{j} \in T_{1}$. Therefore, $\forall j \in J, D_{j} \in T_{1} . \Rightarrow \forall k \in M, D_{k} \in T_{1}$ (as $M \subseteq J$ ). Hence, $\bigwedge_{k \in M} D_{k} \in T_{1}$ (since $T_{1}$ is a theory). And so, $A \in T_{1}$. Therefore, $K \circ_{1} T_{1} \circ_{1} T_{2} \subseteq T_{1}$. Conversely, suppose $A \in T_{1}$. Since $T_{2}$ is not empty, $\exists B$ such that $B \in T_{2}$. By definition, $(A \rightarrow(B \rightarrow A)) \in K$. Therefore, $\mathrm{B} \rightarrow \mathrm{A} \in \mathrm{K} \circ_{1} \mathrm{~T}_{1}$. And hence, $A \in \mathrm{K} \circ_{1} \mathrm{~T}_{1} \circ_{1} \mathrm{~T}_{2}$. Therefore, $\mathrm{T}_{1} \subseteq \mathrm{K} \circ_{1} \mathrm{~T}_{1} \circ_{1} \mathrm{~T}_{2}$ and so, $\mathrm{K} \circ_{1} \mathrm{~T}_{1} \circ_{1} \mathrm{~T}_{2}=\mathrm{T}_{1}$.

Claim i6 $W^{*} \circ_{1} \mathrm{~T}_{1}=\mathrm{T}_{1} \circ_{1} \mathrm{~T}_{1}$.

Proof Suppose $A \in W^{*} \circ_{1} T_{1}$. Then $\exists B$ such that $B \rightarrow A \in W^{*}$ and $B \in T_{1}$. But $B \rightarrow A \in W^{*}$ means that $\exists$ finite I such that $\bigwedge_{i \in I}\left(\left(\left(D_{i} \rightarrow E_{i}\right) \wedge D_{i}\right) \rightarrow\right.$ $\left.E_{i}\right) \leqslant B \rightarrow A$. By the Bubbling Lemma, $\exists J \subseteq$ I such that $B \leqslant \bigwedge_{j \in J}\left(\left(D_{j} \rightarrow\right.\right.$ $\left.\left.E_{j}\right) \wedge D_{j}\right)$ and $\bigwedge_{j \in J} E_{j} \leqslant A$ Now, $\forall j \in J, \bigwedge_{j \in J}\left(\left(D_{j} \rightarrow E_{j}\right) \wedge D_{j}\right) \leqslant\left(\left(D_{j} \rightarrow\right.\right.$ $\left.\left.E_{j}\right) \wedge D_{j}\right)$. Therefore, $\forall j \in J,\left(D_{j} \rightarrow E_{j}\right) \wedge D_{j} \in T_{1}$. So, $\forall j \in J, D_{j} \rightarrow E_{j} \in T_{1}$ and $D_{j} \in T_{1}$. And so, $\forall j \in J, E_{j} \in T_{1} \circ_{1} T_{1}$. Therefore, $\bigwedge_{j \in J} E_{j} \in T_{1}$. And hence, 
$A \in T_{1}$. Therefore, $W^{*} \circ_{1} T_{1} \subseteq T_{1} \circ_{1} T_{1}$. Conversely, suppose $A \in T_{1} \circ_{1} T_{1}$. Then $\exists B$ such that $B \rightarrow A \in T_{1}$ and $B \in T_{1}$. Also $((B \rightarrow A) \wedge B) \rightarrow A \in W^{*}$. But, $(B \rightarrow A) \wedge B \in T_{1}$ (since $T_{1}$ is closed under conjunction). Therefore, $A \in W^{*}$ $\circ_{1} T_{1}$. So, $T_{1} \circ_{1} T_{1} \subseteq W^{*} \circ_{1} T_{1}$. Hence, $W^{*} \circ_{1} T_{1}=T_{1} \circ_{1} T_{1}$.

We have left it to you, dear reader, to verify the defining axioms for the other combinators. But, to be sure that we have a model of CL in Level-I theories, we must show also that truth of the theorems of this system is preserved under the rules. These are succinctly stated in [2, p. 23If].

$$
\begin{aligned}
& \text { (u) } X=Y \Rightarrow U X=U Y \\
& (\nu) X=Y \Rightarrow X U=Y U \\
& (\sigma) \quad X=Y \Rightarrow Y=X \\
& (\tau) \quad X=Y \text { and } Y=Z \Rightarrow X=Z
\end{aligned}
$$

We note simply that, since fusion at Level-I is a single-valued operation on $\mathrm{B} \wedge \mathrm{T}$-theories and since equality is set equality, all of the rules preserve truth, ending the demonstration that there is a model of $C L$ in $B \wedge T$-theories, on any assignment of such theories to free variables.

\section{B $\wedge$ T IS A MODEL FOR COMBINATORS AT LEVEL-II}

Having proved that $\mathrm{B} \wedge \mathrm{T}$-theories provide a good model for the combinators at Level-I, we will now prove the same results at Level-II, i. e., B $\wedge$ T-propositions also provide a good model for the combinators. Let's start with the definitions of the combinators at Level-II. (i) $\iota=\left\{\mathrm{T}_{1}: \mathrm{I} \subseteq \mathrm{T}_{1}\right\}$. (ii) $\mathrm{K}=\left\{\mathrm{T}_{1}: \mathrm{K} \subseteq \mathrm{T}_{1}\right\}$. (iii) $\omega=\left\{T_{1}: W^{*} \subseteq T_{1}\right\}$. Here $\iota, K$ and $\omega$ are the Level-II correspondance of $\mathrm{I}, \mathrm{K}$ and $\mathrm{W}^{*}$ respectively. The definitions of the other combinators follow on similar lines. The idea behind using Greek notations for the combinators at Level-II is just to remove ambiguity with those at Level-I.

Lemma I7 Suppose $T_{1}, T_{2}, T_{3}$ and $T_{4}$ are four theories such that $T_{1} \subseteq T_{2}$ and $T_{3} \subseteq T_{4}$. Then $\mathrm{T}_{1} \circ_{1} \mathrm{~T}_{3} \subseteq \mathrm{T}_{2} \circ_{1} \mathrm{~T}_{4}$.

Proof Suppose $A \in T_{1} \circ_{1} T_{3}$. Then there is some $B$ such that $B \rightarrow A \in T_{1}$ and $\mathrm{B} \in \mathrm{T}_{3}$. Therefore, $\mathrm{B} \rightarrow A \in \mathrm{T}_{2}$ and $\mathrm{B} \in \mathrm{T}_{4}$ (since $\mathrm{T}_{1} \subseteq \mathrm{T}_{2}$ and $\mathrm{T}_{3} \subseteq \mathrm{T}_{4}$ ). Therefore, $A \in \mathrm{T}_{2} \circ_{1} \mathrm{~T}_{4}$. Hence, $\mathrm{T}_{1} \circ_{1} \mathrm{~T}_{3} \subseteq \mathrm{T}_{2} \circ_{1} \mathrm{~T}_{4}$.

Lemma i8 Suppose $\mathrm{P}, \mathrm{Q}, \mathrm{R}$ and $\mathrm{S}$ are four propositions such that $\mathrm{P} \subseteq \mathrm{R}$ and $\mathrm{Q} \subseteq \mathrm{S}$. Then $\mathrm{P} \circ_{2} \mathrm{Q} \subseteq \mathrm{R} \circ_{2} \mathrm{~S}$.

Proof Suppose $\mathrm{T}_{1} \in \mathrm{P} \circ_{2} \mathrm{Q} . \Rightarrow \exists \mathrm{T}_{2} \in \mathrm{P}$ and $\mathrm{T}_{3} \in \mathrm{Q}$ such that $\mathrm{T}_{2} \circ_{1} \mathrm{~T}_{3} \subseteq \mathrm{T}_{1}$. But then, $T_{2} \in R$ and $T_{3} \in S$ (since $P \subseteq R$ and $Q \subseteq S$ ). Therefore, $T_{1} \in R \circ_{2} S$. Hence, $\mathrm{P}_{2} \mathrm{Q} \subseteq \mathrm{R} \circ_{2} \mathrm{~S}$. 
Lemma I9 The fusion of two propositions is also a proposition.

Proof Let $\mathrm{P}, \mathrm{Q}$ be two propositions. (i) Since $\mathrm{P} \neq \emptyset \neq \mathrm{Q}$, it follows that $\exists \mathrm{T}_{1}$ and $T_{2}$ such that $T_{1} \in P$ and $T_{2} \in Q$. Now, $T_{1} \circ_{1} T_{2}$ is a theory (by Lemma Io). And by definition, $T_{1} \circ_{1} T_{2} \in P \circ_{2} Q$. Hence, $P \circ_{2} Q$ is non-empty. (ii) Suppose $\mathrm{T}_{1} \in \mathrm{P} \circ_{2} Q$ and $\mathrm{T}_{1} \subseteq \mathrm{T}_{2}$ and $\mathrm{T}_{2}$ is a theory. So, $\exists \mathrm{T}_{3} \in \mathrm{P}$ and $\mathrm{T}_{4} \in \mathrm{Q}$ where $\mathrm{T}_{3} \circ_{1} \mathrm{~T}_{4} \subseteq \mathrm{T}_{1}$. Therefore $\mathrm{T}_{3} \circ_{1} \mathrm{~T}_{4} \subseteq \mathrm{T}_{2}$ (since $\mathrm{T}_{1} \subseteq \mathrm{T}_{2}$ ). Therefore, $\mathrm{T}_{2} \in \mathrm{P}$ $\circ_{2} Q$. (iii) Suppose $T_{1}, T_{2} \in P \circ_{2} Q$. It follows that $\left(\exists T_{3} \in P\right.$ and $T_{4} \in Q$ where $\left.\left(T_{3} \circ_{1} T_{4} \subseteq T_{1}\right)\right)$ and $\left(\exists T_{5} \in P\right.$ and $T_{6} \in Q$ where $\left.\left(T_{5} \circ{ }_{1} T_{6} \subseteq T_{2}\right)\right)$. Hence, $\left(T_{3} \cap T_{5}\right) \in P$ and $\left(T_{4} \cap T_{6}\right) \in Q$. Also, $\left(T_{3} \cap T_{5}\right) \circ_{1}\left(T_{4} \cap T_{6}\right) \subseteq\left(T_{3} \circ_{1} T_{4}\right) \subseteq$ $\left.T_{1}\right)$ and $\left.\left(T_{3} \cap T_{5}\right) \circ_{1}\left(T_{4} \cap T_{6}\right) \subseteq\left(T_{5} \circ_{1} T_{6}\right) \subseteq T_{2}\right)$ (by Lemma $\left.{ }_{7}\right)$. Therefore, $\left(T_{3} \cap T_{5}\right) \circ_{1}\left(T_{4} \cap T_{6}\right) \subseteq T_{1} \cap T_{2}$. Hence, $T_{1} \cap T_{2} \in P \circ_{2} Q$. Thus, $P \circ_{2} Q$ is non-empty and closed under intersection and sub-theory relation. And hence is a proposition.

THEOREM 20 The fusion of finite number of propositions is a proposition.

Proof By induction on the previous result.

Claim $2 \mathrm{I} 1 \mathrm{O}_{2} \mathrm{P}=\mathrm{P}$.

Proof Suppose $T_{1} \in \iota \circ_{2} P$. It follows that there is some $T_{2} \in \iota$ and $T_{3} \in P$ such that $\mathrm{T}_{2} \circ_{1} \mathrm{~T}_{3} \subseteq \mathrm{T}_{1}$. But $\mathrm{T}_{2} \in \iota \mathrm{\imath} \Rightarrow \mathrm{I} \subseteq \mathrm{T}_{2}$ (by definition). Therefore, I $\circ_{1}$ $\mathrm{T}_{3} \subseteq \mathrm{T}_{2} \circ_{1} \mathrm{~T}_{3}$ (by Lemma I7). Hence, $\mathrm{I} \circ_{1} \mathrm{~T}_{3} \subseteq \mathrm{T}_{1}$. But, I $\circ_{1} \mathrm{~T}_{3}=\mathrm{T}_{3}$ (by Claim I4). $\Rightarrow \mathrm{T}_{3} \subseteq \mathrm{T}_{1}$ and hence, $\mathrm{T}_{1} \in \mathrm{P}$. Therefore, $\iota_{2} \mathrm{O} \subseteq \mathrm{P}$. Conversely, suppose $\mathrm{T}_{1} \in \mathrm{P}$. It follows that $\mathrm{I} \circ_{1} \mathrm{~T}_{1}\left(=\mathrm{T}_{1}\right) \subseteq \mathrm{T}_{1}$. Hence, $\mathrm{T}_{1} \in \iota^{\circ} \circ_{2} \mathrm{P}$. Therefore, $\mathrm{P} \subseteq \iota_{1} \circ_{2} \mathrm{P}$. And so, $\iota_{2} \mathrm{P}=\mathrm{P}$.

Claim $22 \quad \mathrm{~K}_{2} \mathrm{P}_{2} \mathrm{Q}=\mathrm{P}$.

Proof Suppose $T_{1} \in K \circ_{2} P \circ_{2} Q$. So, there is some $T_{2} \in K$, and a $T_{3} \in P$ and $\mathrm{T}_{4} \in \mathrm{Q}$ such that $\mathrm{T}_{2} \circ_{1} \mathrm{~T}_{3} \circ_{1} \mathrm{~T}_{4} \subseteq \mathrm{T}_{1}$. But $\mathrm{T}_{2} \in \mathrm{K} \Rightarrow \mathrm{K} \subseteq \mathrm{T}_{2}$. Therefore, $\mathrm{K} \circ_{1} \mathrm{~T}_{3} \circ_{1}$ $\mathrm{T}_{4} \subseteq \mathrm{T}_{2} \circ_{1} \mathrm{~T}_{3} \circ_{1} \mathrm{~T}_{4} \subseteq \mathrm{T}_{1}$. But $\mathrm{K} \circ_{1} \mathrm{~T}_{3} \circ_{1} \mathrm{~T}_{4}=\mathrm{T}_{3}$ (by Claim 15). $\Rightarrow \mathrm{T}_{3} \subseteq \mathrm{T}_{1}$ and hence $T_{1} \in P$. Therefore, $\mathrm{k} \circ_{2} \mathrm{P} \circ_{2} \mathrm{Q} \subseteq \mathrm{P}$. Conversely, suppose $\mathrm{T}_{1} \in \mathrm{P}$. Since $\mathrm{Q}$ is not empty by definition, there is a $T_{2} \in Q$. $K$ of course belongs to $\mathrm{K}$. Also, $\mathrm{K} \circ_{1} \mathrm{~T}_{1} \circ_{1} \mathrm{~T}_{2}\left(=\mathrm{T}_{1}\right) \subseteq \mathrm{T}_{1}$. Hence, $\mathrm{T}_{1} \in \mathrm{K} \circ_{2} \mathrm{P} \circ_{2} \mathrm{Q}$. and thus $\mathrm{P} \subseteq \mathrm{K} \circ_{2} \mathrm{P} \circ_{2} \mathrm{Q}$. Therefore, $\mathrm{K}_{2} \mathrm{P}_{2} \mathrm{Q}=\mathrm{P}$.

Claim $23 \omega \circ_{2} \mathrm{P}=\mathrm{P}_{\circ_{2}} \mathrm{P}$.

Proof Suppose $T_{1} \in \omega \circ_{2} P$. It follows that there is some $T_{2} \in \omega$ and $T_{3} \in P$ such that $T_{2} \circ_{1} T_{3} \subseteq T_{1}$. But $T_{2} \in \omega \Rightarrow W^{*} \subseteq T_{2}$. Therefore, $W^{*} \circ_{1} T_{3} \subseteq$ $T_{2} \circ_{1} T_{3} \subseteq T_{1}$. But $W^{*} \circ_{1} T_{3}=T_{3} \circ_{1} T_{3}$ (by Claim I6). So $T_{3} \circ_{1} T_{3} \subseteq T_{1}$ and hence $\mathrm{T}_{1} \in \mathrm{P} \circ_{2} \mathrm{P}$. Therefore, $\omega \circ_{2} \mathrm{P} \subseteq \mathrm{P} \circ_{2} \mathrm{P}$. Conversely, suppose $\mathrm{T}_{1} \in \mathrm{P} \circ_{2} \mathrm{P} . \Rightarrow \mathrm{T}_{2} \in \mathrm{P}$ and $\mathrm{T}_{3} \in \mathrm{P}$ such that $\mathrm{T}_{2} \circ_{1} \mathrm{~T}_{3} \subseteq \mathrm{T}_{1}$. But $\mathrm{T}_{2} \in \mathrm{P}$ and $T_{3} \in P \Rightarrow T_{2} \cap T 3 \in P$. $W^{*}$ of course belongs to $\omega$. Also, $W^{*} o_{1}\left(T_{2} \cap T_{3}\right)=$ $\left(T_{2} \cap T_{3}\right) \circ_{1}\left(T_{2} \cap T_{3}\right) \subseteq\left(T_{2} \circ_{1} T_{3}\right) \subseteq T_{1}$. Hence, $T_{1} \in \omega \circ_{2} P$. So $P \circ_{2} P \subseteq \omega \circ_{2} P$. Therefore, $\omega \circ_{2} \mathrm{P}=\mathrm{P} \circ_{2} \mathrm{P}$. 
We conclude this section as we did the last, leaving the verification of other primitive combinator equalities to readers; note that the rules of [2] preserve equality of propositions at Level-II as they did of theories at Level-I.

\section{EXTENSiON OF THE B^T Results TO B+T AT LEVEL-I}

Having shown that $\mathrm{B} \wedge \mathrm{T}$-theories are a model for the combinators at Level-I and $\mathrm{B} \wedge \mathrm{T}$-propositions are a model for the combinators at Level-II, we now try to extend the results to $\mathrm{B}+\mathrm{T}$-theories and propositions. But we are stuck at the very beginning because it is shown in [5] that there is no model for $\lambda$ in the set of all $\mathrm{B}+\mathrm{T}$-theories. For the same reason, there is no model for the combinators there. But we can model the basic combinator laws in prime $\mathrm{B}+\mathrm{T}$ theories, as we proceed to show. Recall that $T_{1}$ is a prime theory if $A \vee B \in T_{1} \Rightarrow$ either $A \in T_{1}$ or $B \in T_{1}$. Again we will talk of only the three combinators $I, K$ and $W^{*}$. The proofs for other primitive combinators from among C, C*, B, B', S and W are similar. For the proofs to go through, we will need what we call the Better Bubbling Lemma (в в ). This is a very important generalization of the Bubbling Lemma в $\mathrm{L}$ above.7 For proofs of вв $\mathrm{b}$, see [7] and [6]. We only state it here.

Lemma 24 (better bubbling lemma) For any finite sets $\left\{\sigma_{i} \rightarrow \tau_{i}\right\}_{i \in M}$ and $\left\{\sigma_{j}^{\prime} \rightarrow \tau_{j}^{\prime}\right\}_{j \in J}$ of arrow types, the following equivalence holds: $\mathfrak{A} \Leftrightarrow \mathfrak{F}$ where

$$
\begin{aligned}
& \mathfrak{A}={ }_{\mathrm{df}} \bigwedge_{i \in M}\left(\sigma_{i} \rightarrow \tau_{i}\right) \leqslant \bigvee_{j \in J}\left(\sigma_{j}^{\prime} \rightarrow \tau_{j}^{\prime}\right) \\
& \mathfrak{F}={ }_{\mathrm{df}} \exists j \in J\left(\sigma_{j}^{\prime} \leqslant \bigvee_{i \in M} \sigma_{i}\right) \text { and } \\
& \qquad \forall M^{\prime} \subsetneq M\left(\sigma_{j}^{\prime} \leqslant \bigvee_{i \in M^{\prime}} \sigma_{i}\right) \text { or }\left(\bigwedge_{i \in M \backslash M^{\prime}} \tau_{i} \leqslant \tau_{j}^{\prime}\right)
\end{aligned}
$$

Proof Given in [6].

From this point on, we will call a $\mathrm{B}+\mathrm{T}$ theory simply a theory; $\mathrm{B}+\mathrm{T}$ proposition, a proposition; and $B+T$ entailment, entailment. We will also use $T_{1}, T_{2}$, etc. to denote theories, $\mathrm{P}, \mathrm{Q}$, etc. to denote propositions, $\underline{A}, \underline{B}$, etc. to denote principal propositions for formulas $A, B$, etc., and $A, B$, etc. to denote well formed formulas. Let us first look at the set of axioms and rules of $B+T$. The axioms are as follows 8

$$
\begin{aligned}
\text { Reflex. } & A \rightarrow A \\
\wedge \mathrm{E} . & (A \wedge B) \rightarrow A,(A \wedge B) \rightarrow B \\
\rightarrow \wedge I . & (A \rightarrow B) \wedge(A \rightarrow C) \rightarrow(A \rightarrow(B \wedge C)) \\
\rightarrow \vee E . & (A \rightarrow C) \wedge(B \rightarrow C) \rightarrow((A \vee B) \rightarrow C) \\
\vee \vee I . & A \rightarrow(A \vee B), B \rightarrow(A \vee B) \\
\text { Dist } \wedge \vee . & A \wedge(B \vee C) \rightarrow((A \wedge B) \vee(A \wedge C))
\end{aligned}
$$

\footnotetext{
${ }^{7}$ It is our impression, based on conversations with Dezani, that BL is mainly due to her and B BL to Castagna.

${ }^{8}$ in contrast to $\mathrm{B} \wedge \mathrm{T}$ above, we formulate $\mathrm{B}+\mathrm{T}$ as an assertional system. But we suppress $\vdash$. To restore it in approved Curry f4 fashion, preface each formula asserted as an axiom with $\vdash$. Make a similar adjustment in the rules.
} 
The rules for the system $B+T$ are as follows. Note that $\Rightarrow$ is again a metalogical connective used to express rules.

$$
\begin{aligned}
\rightarrow \text { E. } & A \rightarrow B \text { and } A \Rightarrow B \\
\wedge \text { I. } & A \text { and } B \Rightarrow A \wedge B \\
\text { RulB. } & B \rightarrow C \Rightarrow(A \rightarrow B) \rightarrow(A \rightarrow C) \\
\text { RulB'. } & A \rightarrow B \Rightarrow(B \rightarrow C) \rightarrow(A \rightarrow C)
\end{aligned}
$$

In what follows, we will show how the prime theories satisfy the combinators. We will show this for the combinators I, K and $\mathrm{W}^{*}$. For the others, the proofs follow on similar lines. Caution: It is necessary to be delicate at this point. What we are proving here (or leaving for you to prove) is that the defining equations for particular primitive combinators are true, when their arguments are assigned prime $\mathrm{B}+\mathrm{T}$ theories. It is also the case that the primitive combinators will be assigned prime theories. Not only that, but any theory all of whose members are entailed by conjunctions of arrow statements will also be prime? This means that prime theories are rather thick on the ground. Nonetheless, as we warn again below, neither the fusion nor the intersection of two prime theories is in general prime. Beware.

Claim 25 Suppose $\mathrm{T}_{1}$ is a prime theory. Then $\mathrm{I}_{1} \mathrm{~T}_{1}=\mathrm{T}_{1}$.

Proof Suppose $A \in I_{1} T_{1}$. So there is a $B$ where $B \rightarrow A \in I$ and $B \in T_{1}$. Now, if $B \rightarrow A \in I$ then there is a finite index set $K$ such that $\bigwedge_{k \in K}\left(C_{k} \rightarrow C_{k}\right) \leqslant B \rightarrow$ A. By Lemma 24, $B \leqslant \bigvee_{k \in K} C_{k}$. But $B \in T_{1}$. Hence, $\bigvee_{k \in K} C_{k} \in T_{1}$. Since $T_{1}$ is prime, there is some $k_{0} \in K$ such that $C_{k_{0}} \in T_{1}$. Define $S=\left\{k \in K: C_{k} \in T_{1}\right\}$. $\left(S \neq \emptyset\right.$ as $k_{0} \in S$.) Define $K^{\prime}=K \backslash S$. $\left(K^{\prime} \subsetneq K\right.$ as $S \neq \emptyset$.) Clearly, $B \not \bigvee_{j \in K^{\prime}} C_{j}$. Therefore, $\bigwedge_{j \in S} C_{j} \leqslant A$. Now, $\forall j \in S, C_{j} \in T_{1}$ (by definition of $S$ ). Hence, $\bigwedge_{j \in S} C_{j} \in T_{1}$ (since $T_{1}$ is a theory). Therefore, $A \in T_{1}$. And so, $I_{\circ} o_{1} \subseteq T_{1}$. Conversely, suppose $A \in T_{1}$. Obviously, $A \rightarrow A \in I$ (by definition). Hence, $A \in I \circ_{1} T_{1}$. Therefore, $\mathrm{T}_{1} \subseteq \mathrm{I}_{1} \mathrm{~T}_{1}$. And so, $\mathrm{I} \circ_{1} \mathrm{~T}_{1}=\mathrm{T}_{1}$.

Claim 26 Suppose $\mathrm{T}_{1}$ and $\mathrm{T}_{2}$ are prime theories. Then $\mathrm{K} \circ_{1} \mathrm{~T}_{1} \circ_{1} \mathrm{~T}_{2}=\mathrm{T}_{1}$.

Proof Suppose $A \in K \circ_{1} T_{1} \circ_{1} T_{2}$. So there are $B, C$ such that $B \rightarrow(C \rightarrow A) \in$ $K, B \in T_{1}$ and $C \in T_{2}$. Now if $B \rightarrow(C \rightarrow A) \in K$ then there is a finite index set $M$ such that $\bigwedge_{i \in M}\left(E_{i} \rightarrow\left(D_{i} \rightarrow E_{i}\right)\right) \leqslant(B \rightarrow(C \rightarrow A))$. By Lemma 24, $B \leqslant \bigvee_{i \in M} E_{i}$. Since, $B \in T_{1}$ and $T_{1}$ is a theory, therefore, $\bigvee_{i \in M} E_{i} \in T_{1}$. Since, $T_{1}$ is prime, there is a $i_{0} \in M$ such that $E_{i_{0}} \in T_{1}$. Define $S=\left\{i \in M: E_{i} \in T_{1}\right\}$ $\left(S \neq \emptyset\right.$ as $\left.i_{0} \in S\right)$. Define $M^{\prime}=M \backslash S\left(M^{\prime} \subsetneq M\right.$ as $\left.S \neq \emptyset\right)$. Clearly, $B \not \bigvee_{j \in M^{\prime}} E_{j}$. Therefore, $\bigwedge_{j \in S}\left(D_{j} \rightarrow E_{j}\right) \leqslant(C \rightarrow A)$. Again by Lemma $24, C \leqslant \bigvee_{j \in S} D_{j}$. Since, $C \in T_{2}$ and $T_{2}$ is a theory, therefore, $\bigvee_{j \in S} D_{j} \in T_{2}$. Since, $T_{2}$ is prime, there is a $j_{0} \in S$ such that $D_{j_{0}} \in T_{2}$. Define $S^{\prime}=\left\{j \in S: D_{j} \in T_{2}\right\}\left(S^{\prime} \neq \emptyset\right.$ as $j_{0} \in S^{\prime}$.) Define $M^{\prime \prime}=S \backslash S^{\prime}\left(M^{\prime \prime} \subseteq S\right.$ as $\left.S^{\prime} \neq \emptyset\right)$. Clearly, $C \nless \bigvee_{j \in M^{\prime \prime}} D_{j}$. Therefore, $\bigwedge_{k \in S^{\prime}} E_{k} \leqslant A$. Now $\forall k \in S^{\prime}, E_{k} \in T_{1}$ (by definition and since

${ }^{9}$ Dezani showed this in [6. 
$S^{\prime} \subsetneq S$ ). Hence, $\bigwedge_{k \in S^{\prime}} E_{k} \in T_{1}$ (since $T_{1}$ is a theory). Therefore, $A \in T_{1}$. And so, $\mathrm{K} \circ_{1} \mathrm{~T}_{1} \circ_{1} \mathrm{~T}_{2} \subseteq \mathrm{T}_{1}$.

Conversely, suppose $A \in T_{1}$. Since $T_{2}$ is not empty, there is some $B$ such that $\mathrm{B} \in \mathrm{T}_{2}$. By definition, $(\mathrm{A} \rightarrow(\mathrm{B} \rightarrow \mathrm{A})) \in \mathrm{K}$. Therefore, $\mathrm{B} \rightarrow \mathrm{A} \in \mathrm{K} \circ_{1} \mathrm{~T}_{1}$. And hence, $A \in K \circ_{1} \mathrm{~T}_{1} \circ_{1} \mathrm{~T}_{2}$. Therefore, $\mathrm{T}_{1} \subseteq \mathrm{K} \circ_{1} \mathrm{~T}_{1} \circ_{1} \mathrm{~T}_{2}$. And so, $\mathrm{K} \circ_{1} \mathrm{~T}_{1} \circ_{1}$ $\mathrm{T}_{2}=\mathrm{T}_{1}$.

Claim 27 Suppose $\mathrm{T}_{1}$ is a prime theory. Then $\mathrm{W}^{*} \circ_{1} \mathrm{~T}_{1}=\mathrm{T}_{1} \circ_{1} \mathrm{~T}_{1}$.

Proof Suppose $A \in W^{*} \circ_{1} T_{1}$. It follows that there is a $B$ such that $B \rightarrow$ $A \in W^{*}$ and $B \in T_{1}$. Now, $B \rightarrow A \in W^{*} \Rightarrow \exists$ a finite index set $M$ such that $\bigwedge_{i \in M}\left(\left(\left(C_{i} \rightarrow D_{i}\right) \wedge C_{i}\right) \rightarrow D_{i}\right) \leqslant B \rightarrow A$. By Lemma 24, $B \leqslant \bigvee_{i \in M}\left(\left(C_{i} \rightarrow\right.\right.$ $\left.\left.D_{i}\right) \wedge C_{i}\right)$. Since, $B \in T_{1}$ and $T_{1}$ is a theory, it follows that, $V_{i \in M}\left(\left(C_{i} \rightarrow\right.\right.$ $\left.\left.D_{i}\right) \wedge C_{i}\right) \in T_{1}$. Since, $T_{1}$ is prime, there is some $i_{0} \in M$ such that $\left(C_{i_{0}} \rightarrow\right.$ $\left.D_{i_{0}}\right) \wedge C_{i_{0}} \in T_{1}$. Define $S=\left\{i \in M:\left(C_{i} \rightarrow D_{i}\right) \wedge C_{i} \in T_{1}\right\}\left(S \neq \emptyset\right.$ as $\left.i_{0} \in S\right)$.

Define $M^{\prime}=M \backslash S\left(M^{\prime} \subsetneq M\right.$ as $\left.S \neq \emptyset\right)$. Clearly, $B \not \bigvee_{j \in M^{\prime}}\left(\left(C_{j} \rightarrow D_{j}\right) \wedge C_{j}\right)$. Therefore, $\bigwedge_{j \in S} D_{j} \leqslant A$. Now, $\forall j \in S,\left(C_{j} \rightarrow D_{j}\right) \wedge C_{j} \in T_{1}$ (by definition of $S$ ). Therefore, $\forall j \in S, C_{j} \rightarrow D_{j} \in T_{1}$ and $C_{j} \in T_{1}$ (since $T_{1}$ is a theory). It follows that $\forall j \in S, D_{j} \in T_{1} \circ_{1} T_{1}$ (by definition). Hence, $\bigwedge_{j \in S} D_{j} \in T_{1} \circ_{1} T_{1}$ (since $T_{1} \circ_{1} T_{1}$ is a theory). Therefore, $A \in T_{1} \circ_{1} T_{1}$. And so, $W^{*} \circ_{1} T_{1} \subseteq T_{1} \circ_{1} T_{1}$.

Conversely, suppose $A \in T_{1} \circ{ }_{1} T_{1}$. Then there is a $B$ such that $B \rightarrow A \in T_{1}$ and $B \in T_{1}$. Also $((B \rightarrow A) \wedge B) \rightarrow A \in W^{*}$. But, $(B \rightarrow A) \wedge B \in T_{1}$ (since $T_{1}$ is closed under conjunction). Therefore, $A \in W^{*} \circ_{1} T_{1}$. So, $T_{1} \circ_{1} T_{1} \subseteq W^{*} \circ_{1} T_{1}$. Hence, $W^{*} \circ_{1} T_{1}=T_{1} \circ_{1} T_{1}$.

\section{THE REAL PROBLEM}

In the above section, we have seen that the prime theories of $B+T$ do a really good job, satisfying all the primitive combinator equalities. So the question arises: Is the set of prime theories of $\mathrm{B}+\mathrm{T}$ a model for the combinators? The answer is "No." We must face the real problem, because prime theories are neither closed under fusion nor closed under intersection 10

So what do we do? The most logical thing to do is to expand a non-prime theory $T_{1}$ to a prime theory. But this can be done in more than one way. So

\footnotetext{
${ }^{\text {Io }}$ Specifically, consider the following counterexample, adapted from Dezani. Recall that we interpret $W=[(A \rightarrow(A \rightarrow B)) \rightarrow(A \rightarrow B)]$, now in the $B+T$ vocabulary. Where $p, q, r$ are propositional variables, define theories $T_{1}$ and $T_{2}$ thus: $T_{1}$ is the principal theory $(p \rightarrow(p \rightarrow$ $r) \wedge(q \rightarrow(q \rightarrow r)) \uparrow$. Similarly let $T_{2}$ be the principal theory $p \vee q \uparrow$. $T_{1}$ is prime, though $T_{2}$ clearly is not prime. Computing, $r \in W \circ_{1} T_{1} \circ_{1} T_{2}$. But $r$ sadly fails to belong to $T_{1} \circ_{1} T_{2} \circ_{1} T_{2}$. This refutes on interpretation in arbitrary $\mathrm{B}+\mathrm{T}$ theories the $\mathrm{W}$ law. Worse, this counterexample can be massaged so that all theories involved are prime. For, where $s$ is another propositional variable, we may simply let $T_{2}=T_{3} \circ_{1} T_{4}$, where $T_{3}$ is the principal theory $s \rightarrow p \vee q \uparrow$ and $T_{4}$ is just $s \uparrow$. The verification that we have left for the reader now fails for $W$, our appeal to B BL being blocked because $T_{2}$, though now defined as a fusion of prime theories, remains resolutely non-prime.
} 
which theory should we choose as our prime extension of $\mathrm{T}_{1}$ ? A natural solution is to look at all the prime theories which are super theories of $T_{1}$. And that is how propositions originate. But in the B+T case we will try to define something more precise, namely, prime propositions. We supply these with a corresponding fusion operator (say, prime fusion) in the hope that the propositions will then have nice properties. So here we go.

Definition 9 (prime proposition) A non-empty collection of theories P is said to be a prime proposition if (i) for each $T_{1} \in P, T_{1}$ is a prime theory. (ii) If $T_{1} \in P, T_{1} \subseteq T_{2}$ and $T_{2}$ is a prime theory, then $T_{2} \in P$.

Definition io (prime fusion operator $\left({ }_{2^{\prime}}\right)$ ) Suppose P and Q are two prime propositions. Then their prime fusion is defined as follows

$$
P \circ_{2^{\prime}} \mathrm{Q}=\left\{\mathrm{T}_{1}:\left(\exists \mathrm{T}_{2} \in \mathrm{P}\right)\left(\exists \mathrm{T}_{3} \in \mathrm{Q}\right)\left(\mathrm{T}_{2} \circ \mathrm{O}_{3}\right) \subseteq \mathrm{T}_{1} \text { and } \mathrm{T}_{1} \text { is prime. }\right\}
$$

THEOREM 28 The intersection of two prime propositions is always non-empty.

Proof Suppose $\mathrm{P}$ and $\mathrm{Q}$ are two prime propositions. By definition, $\mathrm{P} \neq \emptyset \neq$ Q. It follows that there are $T_{1} \in P, T_{2} \in Q$. Now, $T_{1}=\bigcup\left\{C \uparrow: C \in T_{1}\right\}$ And, $T_{2}=\bigcup\left\{D \uparrow: D \in T_{2}\right\}$. Define $T_{3}=\left\{E: \exists C \in T_{1}\right.$ and $\exists D \in T_{2}$ such that $C \wedge D \leqslant$ E\}. Clearly, $T_{1} \subseteq T_{3}$ and $T_{2} \subseteq T_{3}$. However, $T_{3}$ need not be prime. But we can always extend it to some prime theory $T_{4}$ such that $T_{3} \subseteq T_{4}$. Therefore, $T_{4} \in P$ and $\mathrm{T}_{4} \in \mathrm{Q}$, i. e., $\mathrm{T}_{4} \in \mathrm{P} \cap \mathrm{Q}$. And hence, $\mathrm{P} \cap \mathrm{Q}$ is non-empty.

THEOREM 29 The intersection of two prime propositions is always a prime proposition.

Proof Let $\mathrm{P}$ and $\mathrm{Q}$ be two prime propositions. By Theorem 27, $\mathrm{P} \cap \mathrm{Q} \neq \emptyset$. (i) Suppose $T_{1} \in P \cap Q$. Then $T_{1} \in P$ and hence, $T_{1}$ is prime. (ii) Suppose $T_{1} \in P \cap Q$ and $T_{1} \subseteq T_{2}$ and $T_{2}$ is prime. Then $T_{1} \in P, T_{1} \subseteq T_{2}, T_{2}$ is prime and $P$ is a prime proposition. Therefore, $T_{2} \in P$. Similarly $T_{2} \in Q$. And hence $\mathrm{T}_{2} \in \mathrm{P} \cap \mathrm{Q}$. Therefore $\mathrm{P} \cap \mathrm{Q}$ is non-empty, contains only prime theories and is closed under sub-theory relation. And hence is a prime proposition.

THEOREM 30 The prime fusion of two prime propositions is a prime proposition.

Proof Suppose $\mathrm{P}$ and $\mathrm{Q}$ are two prime propositions. (i) Let $\mathrm{T}_{1} \in \mathrm{P} \circ_{2^{\prime}} \mathrm{Q}$. By definition of $\circ_{2^{\prime}}, T_{1}$ is prime. (ii) Since $P \neq \emptyset \neq Q$, there are $T_{1} \in P$ and $T_{2} \in Q$. Now, $T_{1} \circ_{1} T_{2}$ is a theory (by Lemma Io). But it need not be prime. However we can extend it to a prime theory $T_{3}$ such that $T_{1} \circ_{1} T_{2} \subseteq T_{3}$. Then by definition, $\mathrm{T}_{3} \in \mathrm{P}_{2^{\prime}} \mathrm{Q}$. Hence, $\mathrm{P} \circ_{2^{\prime}} \mathrm{Q}$ is non-empty. (iii) Suppose $\mathrm{T}_{1} \in \mathrm{P}_{2^{\prime}} \mathrm{Q}$ and $\mathrm{T}_{1} \subseteq \mathrm{T}_{2}$ and $T_{2}$ is a prime theory. It follows that there are $T_{3} \in P$ and $T_{4} \in Q$ such that $\mathrm{T}_{3} \circ_{1} \mathrm{~T}_{4} \subseteq \mathrm{T}_{1}$. So, $\mathrm{T}_{3} \circ_{1} \mathrm{~T}_{4} \subseteq \mathrm{T}_{2}$. (since $\mathrm{T}_{1} \subseteq \mathrm{T}_{2}$ ) Therefore, $\mathrm{T}_{2} \in \mathrm{P} \circ_{2^{\prime}} \mathrm{Q}$. Thus, $\mathrm{P} \circ_{2^{\prime}}$ Q is non-empty, contains only prime theories and is closed under sub-theory relation. And hence is a prime proposition. 
THEOREM 3I The prime fusion of a finite number of propositions is a prime proposition.

Proof By induction on the previous result.

Prime propositions and the prime fusion operator satisfy the combinator laws for $\mathrm{K}, \mathrm{B}, \mathrm{B}^{\prime}, \mathrm{C}$ and $\mathrm{C}^{*}$. But they do not work so nicely for the combinators $\mathrm{W}, \mathrm{W}^{*}$ and S. We will carry out the arguments for I and K. Proofs for B, B', C and $C^{*}$ are similar. We will indicate where a similar argument breaks down for $W^{*}$. The same difficulties afflict arguments for $W$ and $S$. We begin anew with interpretations of the combinators $\iota, k$ and $\omega$ at Level-II of $B+T . \iota=\left\{T_{1}\right.$ : $\mathrm{I} \subseteq \mathrm{T}_{1}$ and $\mathrm{T}_{1}$ is prime $\} . K=\left\{\mathrm{T}_{1}: \mathrm{K} \subseteq \mathrm{T}_{1}\right.$ and $\mathrm{T}_{1}$ is prime $\} . \omega=\left\{\mathrm{T}_{1}: \mathrm{W}^{*} \subseteq\right.$ $T_{1}$ and $T_{1}$ is prime $\}$.

The theories determined by the combinators are known to be prime [6]. In particular, $I, K$ and $W^{*}$ are prime. Therefore, $I \in \imath, K \in K$ and $W^{*} \in \omega$.

Cla Im 32 Suppose $\mathrm{P}$ is a prime proposition. Then $\iota_{2^{\prime}} \mathrm{P}=\mathrm{P}$.

Proof Suppose $T_{1} \in \iota^{\prime} o_{2}, P$. So $T_{1}$ is prime. It follows that $T_{2} \in \iota$ and $T_{3} \in P$ such that $\mathrm{T}_{2} \circ_{1} \mathrm{~T}_{3} \subseteq \mathrm{T}_{1}$. But $\mathrm{T}_{2} \in \mathrm{\imath} \Rightarrow \mathrm{I} \subseteq \mathrm{T}_{2}$ (by definition). Therefore, $\mathrm{I} \circ_{1} \mathrm{~T}_{3} \subseteq \mathrm{T}_{2} \circ_{1} \mathrm{~T}_{3}$ (by Lemma $\mathrm{I} 7$ ). Hence, $\mathrm{I} \circ_{1} \mathrm{~T}_{3} \subseteq \mathrm{T}_{1}$. But, $\mathrm{T}_{3}$ is prime and so $\mathrm{I} \circ_{1} \mathrm{~T}_{3}=\mathrm{T}_{3}$ (by Claim 25). So $\mathrm{T}_{3} \subseteq \mathrm{T}_{1}$ and hence, $\mathrm{T}_{1} \in \mathrm{P}$ (since $\mathrm{T}_{1}$ is prime). Therefore, $\iota^{\prime} \circ_{2}, P \subseteq P$. Conversely, suppose $T_{1} \in P$. Therefore $T_{1}$ is prime, and $\mathrm{I} \circ_{1} \mathrm{~T}_{1}\left(=\mathrm{T}_{1}\right) \subseteq \mathrm{T}_{1}$. Hence, $\mathrm{T}_{1} \in \iota^{\circ} \circ_{2^{\prime}} \mathrm{P}$. Therefore, $\mathrm{P} \subseteq \iota_{2^{\prime}} \mathrm{P}$. And so, $\iota_{2}, \mathrm{P}=\mathrm{P}$.

Cla 1 m 33 Suppose $\mathrm{P}$ and $\mathrm{Q}$ are prime propositions. Then $\mathrm{k} \circ_{2^{\prime}} \mathrm{P} \circ_{2^{\prime}} \mathrm{Q}=\mathrm{P}$.

Proof Suppose $T_{1} \in \mathrm{K}_{2}{ }_{2} \mathrm{P} \circ_{2^{\prime}} \mathrm{Q}$. Therefore $T_{1}$ is prime. So, there are $T_{2} \in \mathrm{K}$, $\mathrm{T}_{3} \in \mathrm{P}$ and $\mathrm{T}_{4} \in \mathrm{Q}$ such that $\mathrm{T}_{2} \circ_{1} \mathrm{~T}_{3} \circ_{1} \mathrm{~T}_{4} \subseteq \mathrm{T}_{1}$. But if $\mathrm{T}_{2} \in \mathrm{K}$ then $\mathrm{K} \subseteq \mathrm{T}_{2}$. Therefore, $K \circ_{1} \mathrm{~T}_{3} \circ_{1} \mathrm{~T}_{4} \subseteq \mathrm{T}_{2} \circ_{1} \mathrm{~T}_{3} \circ_{1} \mathrm{~T}_{4} \subseteq \mathrm{T}_{1}$. But $\mathrm{T}_{3}$ and $\mathrm{T}_{4}$ are prime. Hence, $K \circ_{1} T_{3} \circ_{1} T_{4}=T_{3}$ (by Claim 26), and $T_{3} \subseteq T_{1}$ which gives $T_{1} \in P$ (since $T_{1}$ is prime). Therefore, $\mathrm{K}_{2^{\prime}} \mathrm{P} \circ_{2^{\prime}} \mathrm{Q} \subseteq \mathrm{P}$.

Conversely, suppose $T_{1} \in P$. Therefore, $T_{1}$ is prime (as $P$ is a prime proposition). Since $Q$ is not empty by definition, there is a $T_{2} \in Q$. $K$ of course belongs to $\mathrm{K}$. Also, $\mathrm{K} \circ_{1} \mathrm{~T}_{1} \circ_{1} \mathrm{~T}_{2}\left(=\mathrm{T}_{1}\right) \subseteq \mathrm{T}_{1}$ and $\mathrm{T}_{1}$ is prime. Hence, $\mathrm{T}_{1} \in \mathrm{K} \circ_{2^{\prime}} \mathrm{P} \circ_{2^{\prime}} \mathrm{Q}$. So, $\mathrm{P} \subseteq \mathrm{K}_{2^{\prime}} \mathrm{P} \circ_{2^{\prime}} \mathrm{Q}$. Therefore, $\mathrm{K} \circ_{2^{\prime}} \mathrm{P} \circ_{2^{\prime}} \mathrm{Q}=\mathrm{P}$.

So we have proved that prime propositions and the prime fusion operator work fine for the combinators I and K. Proofs for the combinators B, B', C and C* are similar. Now we show where we get stuck with these definitions for the combinator $\mathrm{W}^{*}$. Similar difficulties afflict $\mathrm{W}$ and $\mathrm{S}$. We will make the following claim and try to prove it. Keep an eye out for Trouble.

Conjecture 34 Suppose $\mathrm{P}$ is a prime proposition. Then $\omega_{2_{2^{\prime}}} \mathrm{P}=\mathrm{P} \circ_{2^{\prime}} \mathrm{P}$.

Attempted Proof Suppose $T_{1} \in \omega \circ_{2^{\prime}} P$. Hence $T_{1}$ is prime. There are $T_{2} \in \omega$ and $T_{3} \in P$ such that $T_{2} \circ T_{3} \subseteq T_{1}$. But $T_{2} \in \omega \Rightarrow W^{*} \subseteq T_{2}$. Therefore, 
$W^{*} o_{1} T_{3} \subseteq T_{2} \circ_{1} T_{3} \subseteq T_{1}$. But $W^{*} \circ_{1} T_{3}=T_{3} \circ_{1} T_{3}$ (by Claim 27). It follows that $\mathrm{T}_{3} \circ_{1} \mathrm{~T}_{3} \subseteq \mathrm{T}_{1}$ and hence $\mathrm{T}_{1} \in \mathrm{P} \circ_{2^{\prime}} \mathrm{P}\left(\right.$ as $\mathrm{T}_{1}$ is prime). Therefore, $\omega \circ_{2^{\prime}} \mathrm{P} \subseteq \mathrm{P}_{2^{\prime}} \mathrm{P}$.

This part was trivial. It is the other part where we actually get stuck. Conversely, suppose $T_{1} \in P \circ_{2^{\prime}} P$. So, there are $T_{2} \in P$ and $T_{3} \in P$ such that $T_{2} \circ_{1} T_{3} \subseteq T_{1}$. Suppose there is a $T_{4} \in P$ where $T_{4} \subseteq T_{2}$ and $T_{4} \subseteq T_{3}$ [!!!]. $W^{*}$ of course belongs to $\omega$. Also, $W^{*} \circ_{1} T_{4}=\left(T_{4} \circ_{1} T_{4}\right) \subseteq\left(T_{2} \circ_{1} T_{3}\right) \subseteq T_{1}$. Hence, $\mathrm{T}_{1} \in \omega \circ_{2^{\prime}} \mathrm{P}$. It follows that $\mathrm{P} \circ_{2^{\prime}} \mathrm{P} \subseteq \omega \circ_{2^{\prime}} \mathrm{P}$. Therefore, $\omega \circ_{2^{\prime}} \mathrm{P}=\mathrm{P} \circ_{2^{\prime}} \mathrm{P} . b$ Now that the attempted proof is over, look back at the part marked "[!!!]". This line causes a problem. It is not always possible to find a prime theory $T_{4}$ which is a subset of two given arbitrary prime theories $T_{2}$ and $T_{3}$. Consider in this context the boolean algebra on the base set $\{a, b\}$. This lattice illustrates our $B+T$ problems. We have $\{\{a\},\{a, b\}\}$ and $\{\{b\},\{a, b\}\}$ as two prime theories. (Prime filters, as an algebraist would say.) But the only non-empty theory (filter) that is a subset of both of these is $\{\{a, b\}\}$, which unfortunately is not prime. And that is exactly where we get stuck in all the three cases of $\mathrm{W}, \mathrm{W}^{*}$ and $\mathrm{S}$. Perhaps we can modify the definitions of prime propositions and/or the prime fusion operator so that the existence of such a $\mathrm{T}_{4}$ is always guaranteed for arbitrary prime theories $T_{2}$ and $T_{3}$ while satisfying also nice properties like closure under intersection and fusion. If so, we can hope to get a model for the combinators in $\mathrm{B}+\mathrm{T}$-prime propositions. Also note that because of what we have claimed in this paper, any subset of the prime theories of $B+T$, which is closed under intersection and fusion, is definitely a model for $\mathrm{CL}$. But finding one such subset is not an easy goal. We entreat your help.

ACKNOWLEDGEMENT We express our indebtedness to Barendregt, Bunder, Coppo, Dezani, Ghilezan, Hindley, Motohama, Ronchi and Venneri for the substantial discussions Bob had with them on the topics of this paper. The Key to the Universe, the semantical underpinning that links relevant and combinatory logics, grew out of Bob's decades' old collaboration with the late Richard Sylvan.

\section{REFERENCES}

[I] Henk Barendregt, Mario Coppo and Mariangiola Dezani-Ciancaglini, "A Filter Model and the Completeness of Type Assignment", The fournal of Symbolic Logic, Volume 48, Issue 4 (Dec. 1983), 93I-940.

[2] M. W. Bunder, "Combinators, Proofs and Implicational Logics", in D. M. Gabbay and F. Guenther, eds., Handbook of Philosophical Logic, 2nd Edition, Kluwer, Dordrecht (2002), 229-286.

[3] H. B. Curry, and R. Feys, Combinatory Logic vol. I, N. Holland, Amsterdam, (1958).

[4] H. B. Curry, Foundations of Mathematical Logic, McGraw-Hill, N. Y. (1963). 
[5] Mariangiola Dezani-Ciancaglini, Robert K. Meyer and Yoko Motohama "The Semantics of Entailment Omega”, Notre Dame fournal of Formal Logic, vol. 43, no. 3 (2002), I29-I 45 .

[6] Mariangiola Dezani-Ciancaglini, Alain Frisch, Elio Giovannetti and Yoko Motohama, "The Relevance of Semantic Subtyping", Electronic Notes in Theoretical Computer Science 70, No. I (2002).

[7] A. Frisch, G. Castagna and V. Benzaken, "Semantic subtyping", in $17 t h$ IEEE Symposium on Logic in Computer Science, 2002, IEEE Computer Society Press, to appear.

[8] Silvia Ghilezan, "Full Intersection Types and Topologies in Lambda Calculus", fournal of Computer and Science Systems 62 (2OOI), I-I4.

[9] Richard Routley and Robert K. Meyer, "The Semantics of Entailment: III", fournal of Philosophical Logic I (I972), I92-208.

[io] R. Routley, and R. K. Meyer, "The Semantics of Entailment I”, in H. Leblanc, ed., Truth, Syntax and Modality, N. Holland, Amsterdam (1973), I99-243.

[II] M. H. Stone, "The representation theorem for Boolean algebra", Trans. Am. Math. Soc. 40 (I936), 37-III. 
The Australasian fournal of Logic (ISSN I448-5052) disseminates articles that significantly advance the study of logic, in its mathematical, philosophical or computational guises. The scope of the journal includes all areas of logic, both pure and applied to topics in philosophy, mathematics, computation, linguistics and the other sciences.

Articles appearing in the journal have been carefully and critically refereed under the responsibility of members of the Editorial Board. Only papers judged to be both significant and excellent are accepted for publication.

The journal is freely available at the journal website at

$$
\text { http://www.philosophy.unimelb.edu.au/ajl/ }
$$

All issues of the journal are archived electronically at the journal website.

SuBSCRIPTIONS Individuals may subscribe to the journal by sending an email, including a full name, an institutional affiliation and an email address to the managing editor at ajl-editors@unimelb.edu.au Subscribers will receive email abstracts of accepted papers to an address of their choice. For institutional subscription, please email the managing editor at ajl-editors@unimelb.edu.au.

Complete published papers may be downloaded at the journal's website at http: //www.philosophy.unimelb.edu.au/ajl/ The journal currently publishes in pdf format.

Submission The journal accepts submissions of papers electronically. To submit an article for publication, send the $\mathrm{LT}_{\mathrm{E}} \mathrm{X}$ source of a submission to a member of the editorial board. For a current list of the editorial board, consult the website.

The copyright of each article remains with the author or authors of that article. 